\title{
Inhibition of FOXQ1 induces apoptosis and suppresses proliferation in prostate cancer cells by controlling BCL11A/MDM2 expression
}

\author{
XIANG ZHANG ${ }^{1,2}$, LIJUAN WANG ${ }^{3}$, YINGMEI WANG ${ }^{4}$, SHENJIA SHI $^{5}$, HUAYU ZHU $^{6}$, FENGJIN XIAO ${ }^{1}$, \\ JING YANG $^{1}$, ANGANG YANG $^{2}$ and XIAOKE HAO ${ }^{1}$ \\ ${ }^{1}$ Department of Clinical Laboratory Medicine, Xijing Hospital, Fourth Military Medical University, Xi'an, Shaanxi 710032; \\ ${ }^{2}$ Department of Immunology, Basic Medical College, Fourth Military Medical University, Xi'an, Shaanxi 710032; \\ ${ }^{3}$ Department of Oncology, The First Affiliated Hospital, College of Medicine, Xi'an Jiaotong University, Xi'an, \\ Shaanxi 710032; ${ }^{4}$ Department of Pathology, Xijing Hospital, Fourth Military Medical University, Xi'an, Shaanxi 710032; \\ ${ }^{5}$ Department of Aristogenesis, No. 202 Hospital of PLA, Shenyang, Liaoning 110000; ${ }^{6}$ Department of Burns \\ and Cutaneous Surgery, Xijing Hospital, Fourth Military Medical University, Xi'an, Shaanxi 710032, P.R. China
}

Received February 3, 2016; Accepted March 15, 2016

DOI: $10.3892 / o r .2016 .5018$

\begin{abstract}
Forkhead box Q1 (FOXQ1) has been recognized as an oncogene that is overexpressed in different cancers, and several studies have shown that FOXQ1 is related to apoptosis and proliferation in many cancer types. However, the role and the molecular mechanism of FOXQ1 in prostate cancer remains unclear. In this study, we aimed to explore the role of FOXQ1 in regulating cell apoptosis, proliferation and invasion in prostate cancer and the underlying mechanism. We found that FOXQ1 was highly expressed in the prostate cancer tissues and cell lines. In our FOXQ1 loss-of-function experiments, the data indicate that the expression of BCL11A and MDM2 was significantly downregulated, prostate cancer cell proliferation and invasion were markedly suppressed, and apoptosis was significantly induced. Moreover, overexpression
\end{abstract}

Correspondence to: Dr Angang Yang, Department of Immunology, Basic Medical College, Fourth Military Medical University, 15 West Changle Road, Xi'an, Shaanxi 710032, P.R. China

E-mail: angangyangat@sina.com

Dr Xiaoke Hao, Department of Clinical Laboratory Medicine, Xijing Hospital, Fourth Military Medical University, 15 West Changle Road, Xi'an, Shaanxi 710032, P.R. China

E-mail: xiaokehaoman@163.com

Abbreviations: FOXQ1, forkhead box Q1; BCL, B cell lymphoma/ leukemia; MDM2, murine double minute 2; FBS, fetal bovine serum; SDS-PAGE, sodium dodecyl sulfate-polyacrylamide gel electrophoresis; Annexin V-FITC/PI, Annexin V-fluorescein isothiocyanate/propidium iodide; MTT, 3-(4,5-dimethylthiazol-2-yl)2,5-diphenyltetrazolium bromide; BrdU, bromodeoxyuridine

Key words: cell proliferation, prostate cancer, forkhead box Q1, BCL11A, MDM2, cell apoptosis of BCL11A obviously reversed the effect of FOXQ1 inhibition on apoptosis, proliferation and invasion of prostate cancer cells. In addition, BCL11A overexpression also abrogated the inhibitory effect of FOXQ1 suppression on MDM2 expression. Taken together, our study suggests that FOXQ1 regulates prostate cancer cell proliferation and apoptosis by regulating BCL11A/MDM2 expression and indicates that FOXQ1 may serve as a potential therapeutic target for prostate cancer.

\section{Introduction}

Prostate cancer is the most common male genitourinary system malignancy, and causes major public health problems worldwide $(1,2)$. The incidence of prostate cancer in older men is rapidly increasing every year (3). Cancer often compromises adjacent tissues and organs during the development of the disease and can result in a wide range of bone metastases, seriously affecting the survival and quality of life of patients. Thus far, the underlying molecular mechanism of prostate cancer remains unclear. To this end, it is necessary to investigate the biological mechanism driving prostate cancer to provide novel insight into the prevention and therapeutic management of this disease.

Forkhead box Q1 (FOXQ1) is a transcription factor that exerts its biological effects by controlling gene transcription activity (4-6). FOXQ1 encodes a forkhead box (FOX) family related protein (6-8). The FOX family is involved in the regulation of various biological activities, such as development $(7,9)$, metabolism (10), cancer (11), and aging (12). In recent years, the role of FOXQ1 as a proto-oncogene has gradually been recognized, and it is found in colon cancer (7), ovarian cancer (13), breast cancer $(7,14)$, nasopharyngeal carcinoma (15), glioma (16), non-small cell lung cancer (NSCLC) (17) and other tumors. Moreover, FOXQ1 plays a role in proliferation (18), drug resistance (19), epithelial-mesenchymal transition (EMT) (19), angiogenesis (17) and metastasis (20) in cancer. It has been reported that FOXQ1 is upregulated in 
colorectal cancer, inhibiting apoptosis and accelerating tumor growth (21). Moreover, FOXQ1 has a significant function in tumor growth in bladder cancer (22). FOXQ1 promotes the invasive ability of laryngeal carcinoma (23) and enhances the metastasis of hepatocellular carcinoma and non-small cell lung cancer by regulating EMT $(4,17)$. However, the role of FOXQ1 in prostate cancer remains poorly understood.

BCL (B cell lymphoma/leukemia) 11A is a member of the $\mathrm{BCL}$ family, and is a transcription factor that negatively regulates fetal hemoglobin generation (24). BCL11A is expressed in the bone marrow, spleen, B and T cells, monocytes and megakaryocytes (25). Additionally, BCL11A is related to the development of $\mathrm{B}$ cell malignancies, in which it is expressed at high levels (26). BCL11A is upregulated by FOXQ1 overexpression in colorectal cancer and acute myeloid leukemia $(21,27)$.

Studies have indicated that BCL11A regulates the expression of murine double minute 2 (MDM2) $(25,28)$. MDM2 encodes for a protein that acts as a major regulator of the tumor-suppressor gene p53, and is involved in the regulation of cell growth, apoptosis and the cell cycle (29,30). MDM2 is overexpressed in human gastric cancer, bladder cancer and sarcoma, and expressed at lower levels in the corresponding normal tissues, suggesting that MDM2 plays a role in the course of cancer development (31-33). It has been indicated that MDM2 inhibition induces apoptosis in hepatoma and constrains the proliferation and invasion of these cells (34). Moreover, studies have indicated that MDM2 is a potential target for prostate cancer therapy, as downregulation of MDM2 suppresses prostate cancer cell proliferation and metastasis and promotes apoptosis (35).

In this study, we aimed to investigate the role of FOXQ1 in regulating prostate cancer cell proliferation and apoptosis and its potential relationship with BCL11A and MDM2. We found that FOXQ1 is overexpressed in prostate cancer tissues and cell lines and the inhibition of FOXQ1 induces cells apoptosis and suppresses the proliferation and invasion of prostate cancer cells. Moreover, the data demonstrate that FOXQ1 may play a role in regulating MDM2 by controlling BCL11A in prostate cancer. Our study suggests that FOXQ1 plays an important role in prostate cancer and provided a novel insight into preventing and treating prostate cancer through targeting DOXQ1.

\section{Materials and methods}

Cell lines. Human prostate cancer cell lines (PC-3, DU-145 and $\mathrm{LNCaP}$ ) were purchased from the Cell Bank of the Chinese Academy of Sciences (Shanghai, China). These cell lines were cultured in RPMI-1640 with $10 \%$ fetal bovine serum (FBS) (both from Hyclone, Salt Lake City, UT, USA) and $1 \%$ penicillin/streptomycin (Gibco, Rockville, MD, USA). Additionally, the human non-neoplastic prostatic epithelial RWPE-1 obtained from the American Type Culture Collection (ATCC; Rockwell, MD, USA) were maintained in keratinocyte serum-free medium (Gibco) containing $50 \mu \mathrm{g} / \mathrm{ml}$ of bovine pituitary extract, $0.5 \%$ penicillin-streptomycin mix, and $5 \mathrm{ng} / \mathrm{ml}$ epidermal growth factor. The cell lines were cultured in a humidified atmosphere containing $5 \% \mathrm{CO}_{2}$ at $37^{\circ} \mathrm{C}$.

Tissue specimens. Tumor tissues and matched adjacent normal tissues were obtained from Fourth Military Medical University and tissues were cryopreserved immediately following laparoscopic radical prostatectomy. The set of prostate tissues included 10 tumor tissues and 10 matched adjacent normal tissues. The study was approved by the hospital ethics board.

Real-time quantitative polymerase chain reaction ( $R T-q P C R)$ assay. Total RNA was extracted from cell lines using TRIzol (Thermo Fisher Scientific, Waltham, MA, USA). The method of total RNA extraction from tissues was performed according to the literature (36). Subsequently, the total RNA was synthesized into cDNA using the RevertAid First Strand cDNA Synthesis kit (Thermo Fisher Scientific) as per the protocol. All of the RT-qPCR reactions used $20 \mu 1$, with $10 \mu \mathrm{l}$ of SYBR Green PCR Master Mix (Applied Biosystems, Carlsbad, CA, USA) and primers as follows: FOXQ1 forward, 5'-ATTTCTTG CTATTGACCGATGC-3' and reverse, 5'-CCCAAGGAGACC ACAGTTAGAG-3'; GAPDH forward, 5'-GGAAGATGGTGA TGGGATT-3' and reverse, 5'-GGATTTGGTCGTATTGGG-3'. E-cadherin forward, 5'-GGCTGCGGCTGCAGAGACTGG-3' and reverse, 5'-TACACTGCCCAGGAGCCAGA-3'; and vimentin forward, 5'-TGAGTACCGGAGACAGGTGCAG-3' and reverse, 5'-GGAGCCACTGCCTTCATAGTCAA-3'. The relative levels of gene expression were estimated by the $2^{-\Delta \Delta \mathrm{Ct}}$ method.

Western blot analysis. Total proteins were extracted from the cell lines using lysis buffer (Beyotime, Nantong, China) and phenylmethanesulfonyl fluoride (100:1). The concentrations of proteins were measured using a BCA kit (Beyotime), and the equivalent volume containing $25 \mu \mathrm{g}$ of protein was separated by $12 \%$ sodium dodecyl sulfate-polyacrylamide gel electrophoresis (SDS-PAGE). The proteins were then transferred to a nitrocellulose membrane using a semi-dry blotting apparatus (both from Bio-Rad, Hercules, CA, USA). The membrane was blocked in 5\% skim milk diluted in Tris-buffered saline at room temperature for $2 \mathrm{~h}$ and then incubated with primary antibodies against anti-FOXQ1 (1:500), anti-BCL11A (1:800), anti-MDM2 (1:500), anti-E-cadherin (1:500), anti-vimentin $(1: 500)$ and anti-GAPDH $(1: 1,000)$ purchased from Santa Cruz Biotechnology Inc. (Santa Cruz, CA, USA) overnight at $4^{\circ} \mathrm{C}$. The membrane was blotted with a horseradish peroxidase-conjugated secondary antibody (Cell Signaling Technology, Danvers, MA, USA; 1:1,000) for $2 \mathrm{~h}$ followed by washing with Tris-buffered saline with Tween (TBST). Finally, the target proteins were analyzed using a Bio-Rad ChemiDoc apparatus, and the signal intensity was measured by Image-Pro Plus 6.0 software.

Caspase-3 activity assay. Caspase-3 activity assay in the cells was assessed on the basis of the standard instructions of a Caspase-3 activity assay kit (Beyotime).

Annexin-V fluorescein isothiocyanate conjugate and propidium iodide Annexin V (Annexin V-FITC/PI). Apoptosis was assessed according to the manufacturer's instructions in the Annexin V-FITC Apoptosis detection kit (Bender MedSystems, Vienna, Austria). The cells $\left(5 \times 10^{5}\right.$ cells $\left./ \mathrm{ml}\right)$ were resuspended in the binding buffer diluted in distilled deionized water (1:4) and mixed with $5 \mu$ l of Annexin V-FITC in $195 \mu \mathrm{l}$ of the cell suspension. The mixture was then 
incubated at room temperature for $10 \mathrm{~min}$, then washed in phosphate-buffered saline. Thereafter, the cells were suspended in $190 \mu \mathrm{l}$ of diluted binding buffer and $10 \mu \mathrm{l}$ of PI was added. Apoptotic cells were counted using a FACS analyzer (Beckman Coulter, Kraemer Boulevard Brea, CA, USA).

Measurement of cell growth and viability. Cell growth and viability were tested using the 3-(4,5-dimethylthiazol-2-yl)2,5-diphenyltetrazolium bromide (MTT) assay. The cells were plated in 96-well plates and cultured in an incubator with $5 \%$ $\mathrm{CO}_{2}$ at $37^{\circ} \mathrm{C}$. A total of $25 \mu \mathrm{l} \mathrm{MTT}(5 \mathrm{~g} / \mathrm{l})$ was added after removing the cell culture medium and allowed to react for $5 \mathrm{~h}$ at $37^{\circ} \mathrm{C}$. Afterwards, the crystals were dissolved in $150 \mu \mathrm{l}$ of dimethyl sulfoxide. The results were measured using an ELISA reader (Titertek Plus MS 212; ICN, Eschwege, Germany) at a wavelength of $490 \mathrm{~nm}$. All experiments were performed in triplicate.

Bromodeoxyuridine (BrdU) assay. Cell proliferation was assessed by a BrdU cell proliferation assay kit (Millipore, Billerica, MA, USA). The protocols were performed as per the instructions. Cells were plated in 96-well plates and $10 \mu \mathrm{l}$ of BrdU solution was added per well and allowed to react for $1 \mathrm{~h}$. The culture medium was replaced by denaturing solution (100 $\mu \mathrm{l} /$ well) and incubated for $20 \mathrm{~min}$. Thereafter, the detection antibody solution was added and incubated for $1.5 \mathrm{~h}$ at room temperature. The secondary antibody solution $(100 \mu \mathrm{l} /$ well) was added following three washes with wash buffer. Next, $100 \mu 1$ tetramethylbenzidine (TMB) substrate was added and allowed to incubate for $25 \mathrm{~min}$. The DNA incorporation of BrdU was detected at $450 \mathrm{~nm}$ on a SpectroFluor Plus Multiwell plate reader (Tecan, Research Triangle Park, NC, USA). All experiments were performed in triplicate.

Cell invasion assay. Prostate cancer cell invasion ability was detected using Bio-Coat cell migration chambers (BD Biosciences, San Jose, CA, USA). Filters (Corning Incorporated, Toledo, NY, USA) were coated with Matrigel (Becton Dickinson, Bedford, MA, USA). The cells were resuspended in $300 \mu \mathrm{l}$ of serum-free medium ( $2 \times 10^{5}$ cells) and added to the insert (top chamber). A total of $500 \mu \mathrm{l}$ of culture medium with $10 \%$ FBS was then added to the lower chamber and cultured in an incubator with $5 \% \mathrm{CO}_{2}$ at $37^{\circ} \mathrm{C}$ for $48 \mathrm{~h}$. Non-invading cells were gently removed by a cotton swab, and invasive cells were fixed, stained and observed using a microscope. The number of invaded cells was counted in six random fields per membrane and averaged. Each assay was repeated three times.

Construction of recombinant plasmids. Full-length human BCL11A (NM_022893.3) was amplified using the reverse transcription polymerase chain reaction and then subcloned into the plasmid pcDNA.3.1/myc-His(-)Avector (Invitrogen, Carlsbad, CA, USA). The restriction sites of BCL11A cDNA and pcDNA.3.1/myc-His(-)Avector were EcoRI and BamHI. Next, the transformation of recombined plasmids into $E$. coli DH5 $\alpha$ (Tiangen, Beijing, China) was conducted, and the recombined plasmids were amplified overnight at $37^{\circ} \mathrm{C}$.

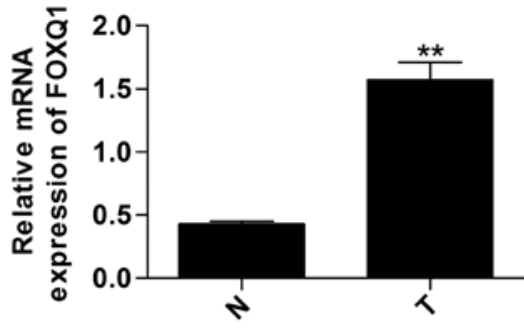

Figure 1. Relative mRNA expression of FOXQ1 in prostate cancer tissues. Relative mRNA expression of FOXQ1 was measured using RT-qPCR GAPDH was used as the internal control. $\mathrm{N}$, matched adjacent normal tissues; $\mathrm{T}$, tumor tissues. $\mathrm{n}=10,{ }^{* *} \mathrm{P}<0.01$ vs. the $\mathrm{N}$ group indicates a significant difference.

Finally, the amplified plasmids were extracted and sequenced, and designated as pcDNA.3.1/-BCL11A.

Cell transfection. The PC-3 $\left(3 \times 10^{4}\right.$ cells/well) and DU-145 $\left(3 \times 10^{4}\right.$ cells/well) cells were plated in 24 -well plates, separately. Transfection of FOXQ1 small-interfering RNA (siRNA, 5'-CGCGGACTTTGCACTTTGA-3'), non-specific siRNA (5'-TTCTCCGAACGTGTCACGT-3') or co-transfection of pcDNA.3.1/-BCL11A and FOXQ1 siRNA was performed using Turbofect (Thermo Fisher Scientific) in accordance with the manufacturer's instructions. The transfected cells were maintained in an incubator (Thermo Fisher Scientific) with 5\% $\mathrm{CO}_{2}$ at $37^{\circ} \mathrm{C}$ for $48 \mathrm{~h}$, and the transfection efficiency was measured by RT-qPCR and western blot analyses.

Statistical analysis. The statistical analyses were performed to test for statistically significant differences between two groups using Student's t-test and between multiple groups using one-way ANOVA. Data are expressed as the mean \pm standard deviation (SD). A p-value of $<0.05$ was considered statistically significant.

\section{Results}

High expression of FOXQ1 in prostate cancer tissues and cell lines. To investigate the expression of FOXQ1 in prostate cancer, we tested the relative mRNA expression using RT-qPCR in tumor tissues and matched adjacent normal tissues. The data show that the mRNA expression of FOXQ1 was significantly higher in tumor tissue than in the matched adjacent normal tissues (Fig. 1). Furthermore, FOXQ1 mRNA and protein expression in prostate cancer cell lines, including PC-3, DU-145 and LNCaP, were measured by RT-qPCR and western blot analysis, respectively. The results show that the mRNA (Fig. 2A) and protein (Fig. 2B) expression of FOXQ1 in prostate cancer lines (PC-3, DU-145 and LNCaP) was obviously upregulated compared with the non-neoplastic cell line RWPE-1. These results indicate that FOXQ1 may play an important role in prostate cancer.

FOXQ1 inhibition constrains the expression of BCL11A and $M D M 2$. To explore the influence of FOXQ1 on BCL11A and MDM2, we performed a FOXQ1 loss-of-function experiment in PC-3 and DU-145 cells by transfection with siRNA targeted to FOXQ1. The results show that the mRNA (Fig. 3A) and 
A

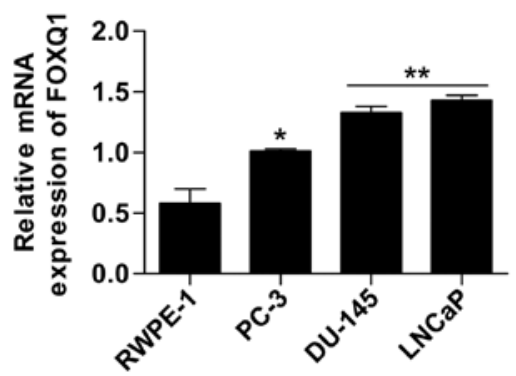

B
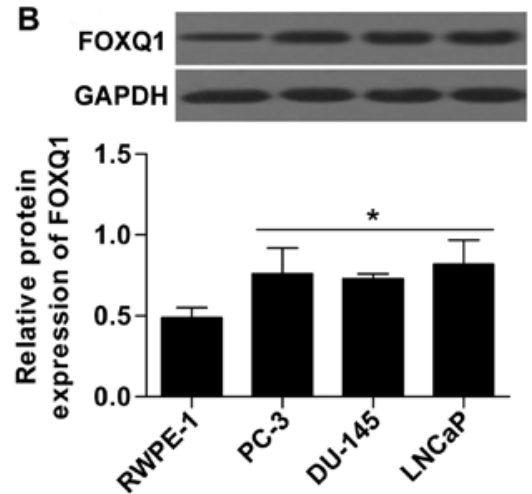

Figure 2. Expression of FOXQ1 in prostate cancer cell lines. The relative mRNA level (A) and protein level (B) of FOXQ1 were measured by qRT-PCR and western blot analyses, respectively. GAPDH was used as the internal control. PC-3, DU-145 and LNCaP are prostate cancer cell lines while RWPE-1 is a non-neoplastic cell line. $n=3,{ }^{*} \mathrm{P}<0.05$ and ${ }^{* *} \mathrm{P}<0.01$ vs. RWPE-1 cells indicate a significant difference. The relative protein expression of FOXQ1 in each cell group was detected using Image-Pro Plus 6.0.

A
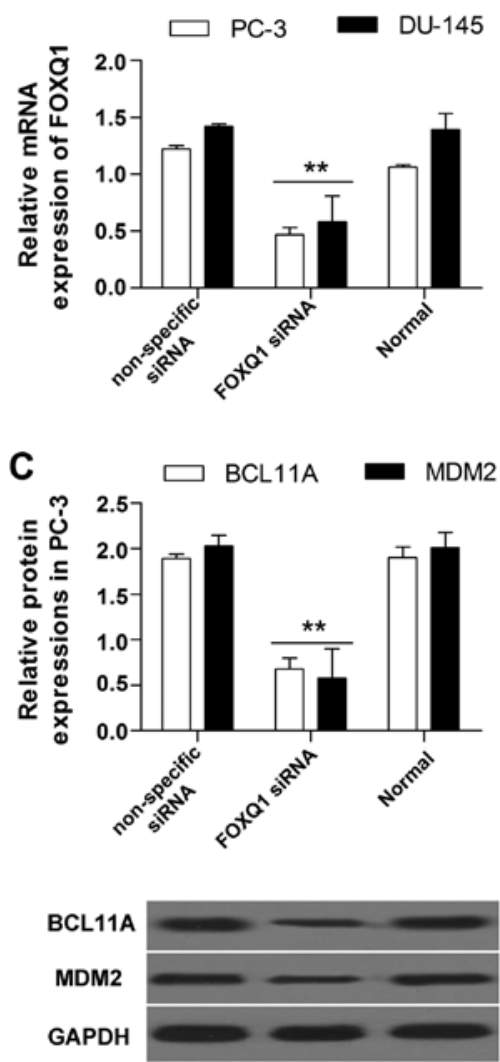

B
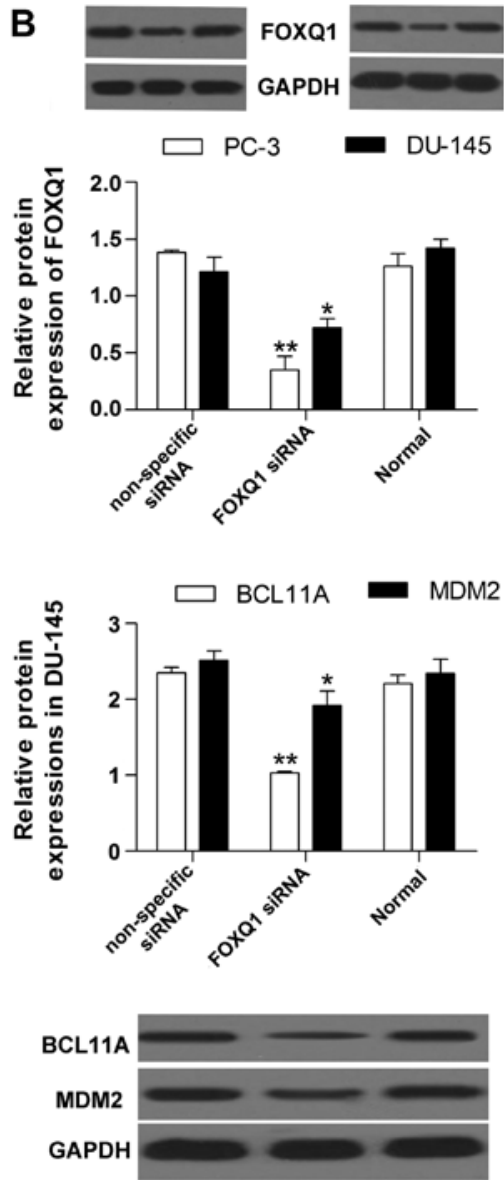

Figure 3. Expression of BCL11A and MDM2 after FOXQ1 inhibition. The relative mRNA level (A) and protein level (B) of FOXQ1 were measured by the qRT-PCR and western blot analyses in PC-3 and DU-145 cell lines. The relative protein levels of BCL11A and MDM2 were measured by western blot analysis in PC-3 (C left) and DU-145 (C right) cell lines. Non-specific siRNA, cells transfected with non-specific siRNAs; FOXQ1 siRNA, cells transfected with FOXQ1 siRNAs; normal, PC-3 or DU-145 cell line without transfection. The GAPDH was used as the control. $\mathrm{n}=3,{ }^{*} \mathrm{P}<0.05$ and ${ }^{* *} \mathrm{P}<0.01$ vs. the non-specific siRNA group. The relative protein expression in each cell group was detected using Image-Pro Plus 6.0.

protein (Fig. 3B) expression of FOXQ1 were both markedly decreased by FOXQ1 siRNA, as determined by RT-qPCR and western blot analysis, indicating that FOXQ1 inhibition was successful. Moreover, we found that FOXQ1 inhibition significantly decreased the protein expression of BCL11A and MDM2 (Fig. 3C) in prostate cancer cells.
FOXQ1 inhibition induces prostate cancer cell apoptosis. Next, we tested the effect of FOXQ1 inhibition on apoptosis in PC-3 and DU-145 cells using the Annexin V-FITC/PI assay and by assessing caspase-3 activity. In the Annexin V-FITC/PI assay, apoptosis was markedly increased by FOXQ1 siRNA (Fig. 4A). Furthermore, the results show that caspase- 3 activity was 
A

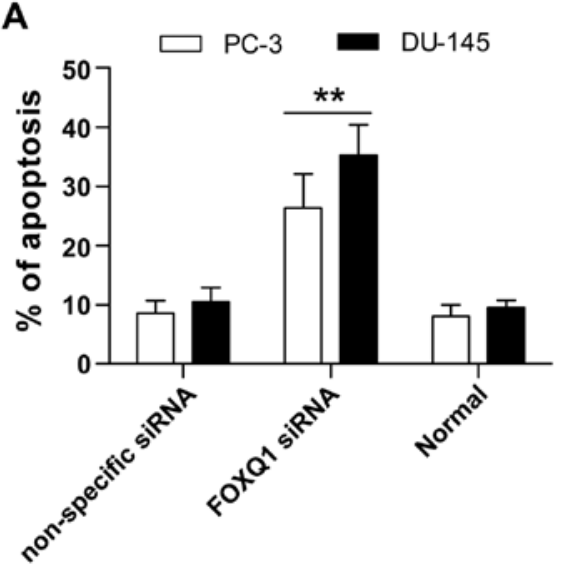

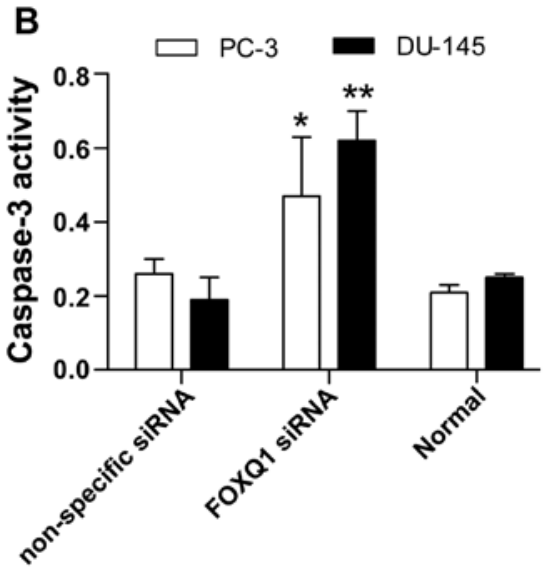

Figure 4. Effect of FOXQ1 suppression on apoptosis in prostate cancer cells. Apoptosis of PC-3 and DU-145 cells was assessed by the Annexin V-FITC/PI assay (A) and caspase-3 activity (B) in cells treated with non-specific siRNA or FOXQ1 siRNA for $48 \mathrm{~h}$. ${ }^{*} \mathrm{P}<0.05$ and ${ }^{* *} \mathrm{P}<0.01$ vs. the non-specific siRNA group.
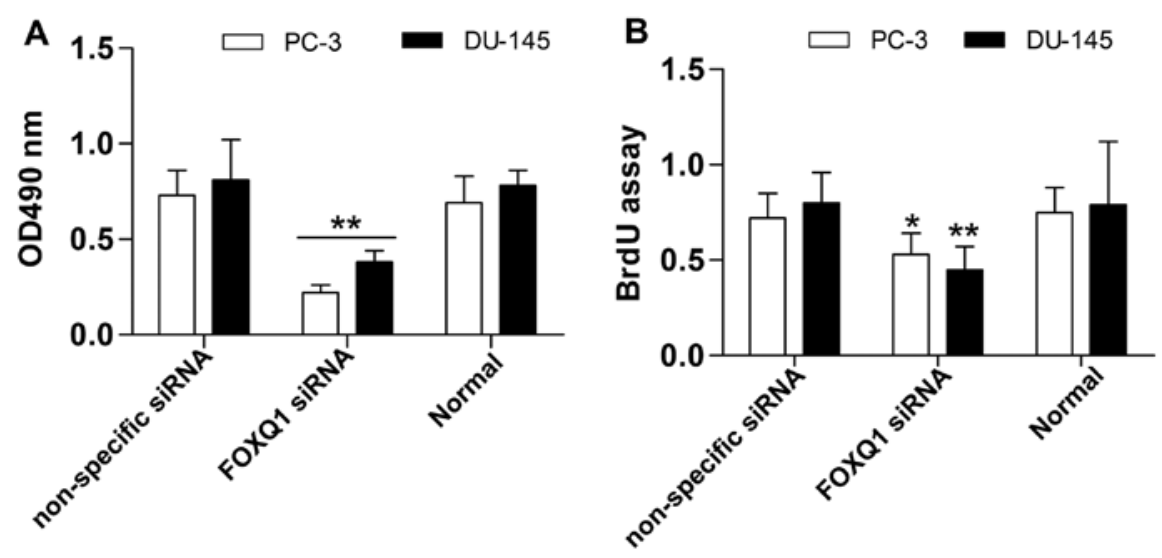

Figure 5. Effect of FOXQ1 suppression on proliferation in prostate cancer cells. The proliferation of cell lines was measured by the MTT assay (A) and BrdU assay (B) in cells treated with non-specific siRNA or FOXQ1 siRNA for $48 \mathrm{~h}$. ${ }^{*} \mathrm{P}<0.05$ and ${ }^{* *} \mathrm{P}<0.01$ vs. the non-specific siRNA group.

greatly increased by FOXQ1 inhibition (Fig. 4B). These results demonstrate that suppression of FOXQ1 promotes apoptosis in prostate cancer cells.

Suppression of FOXQ1 inhibits prostate cancer cell proliferation and invasion. To further investigate the biological effect of FOXQ1 inhibition in prostate cancer cells, we measured its effect on prostate cancer cell proliferation and invasion. The results show that prostate cancer cell proliferation was significantly restrained by FOXQ1 inhibition, as detected by the MTT (Fig. 5A) and BrdU (Fig. 5B) assays. Moreover, the Transwell invasion assay showed that FOXQ1 suppression significantly restrained the invasion ability of prostate cancer cells (Fig. 6A). Western blot analysis indicated that FOXQ1 suppression markedly increased E-cadherin expression and inhibited vimentin expression in prostate cancer cells (Fig. 6B). Above all, these results suggest that FOXQ1 inhibition suppresses the proliferation and invasion of prostate cancer cells.

Inhibition of FOXQ1 constrains MDM2 by controlling BCL11A expression. To investigate the potential molecular mechanism of FOXQ1 in prostate cancer, we performed co-transfection of FOXQ1 siRNA with the recombinant plasmid pcDNA.3.1/-BCL11A. The western blot analysis showed that upregulation of BCL11A significantly reversed the inhibitory effect of FOXQ1 siRNA on BCL11A expression (Fig. 7A and B). Furthermore, the decreased expression of MDM2 induced by FOXQ1 siRNA was also significantly reversed by BCL11A overexpression (Fig. 7A and B). Additionally, the induction of apoptosis (Fig. 8A) and inhibition of proliferation (Fig. 8B) and invasion (Fig. 8C) caused by FOXQ1 suppression were all obviously reversed by BCL11A overexpression.

\section{Discussion}

As a malignant tumor, the morbidity of prostate cancer is increasing and the therapy for this disease is still a challenge (37). In recent years, the FOXQ1 oncogene has attracted increasing attention in the cancer field. Studies have shown that FOXQ1 is a transcription factor that plays a role in cancer metastasis and development (38). It has been reported that high expression of FOXQ1 is found in cancers such as NSCLC, colorectal cancer, breast cancer, glioma, and hepatocellular carcinoma (39). FOXQ1 overexpression enhances invasion and metastasis by promoting EMT activity in bladder cancer and 

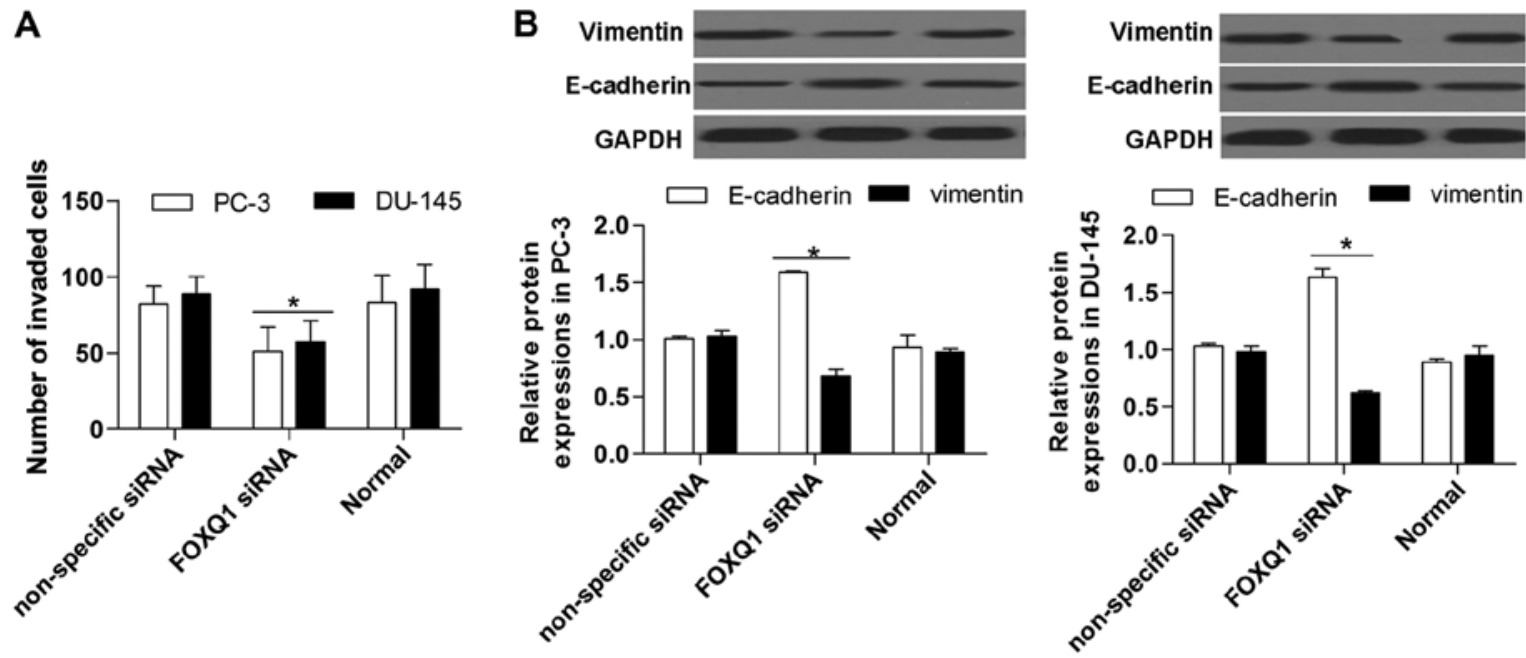

Figure 6. Effects of FOXQ1 suppression on invasion and EMT in prostate cancer cells. Invasion and EMT were assessed in prostate cancer cells using the Transwell invasion assay (A) and western blot analysis (B), respectively. ${ }^{*} \mathrm{P}<0.05$ vs. the non-specific siRNA group.

A

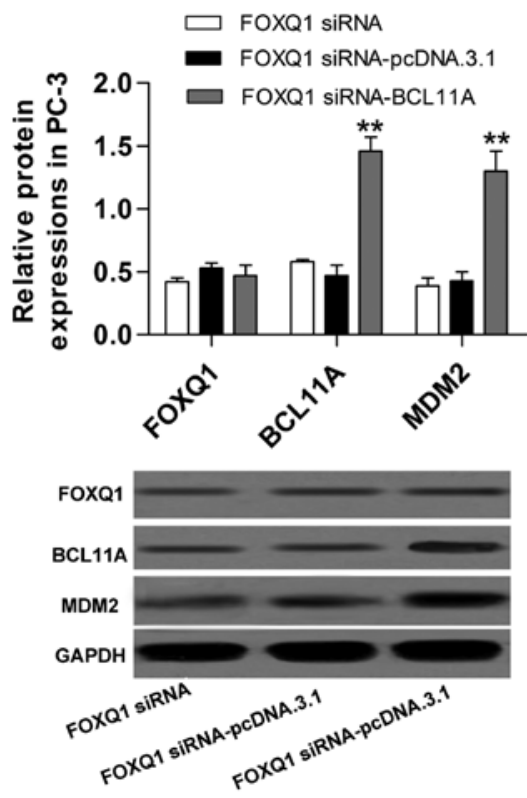

B

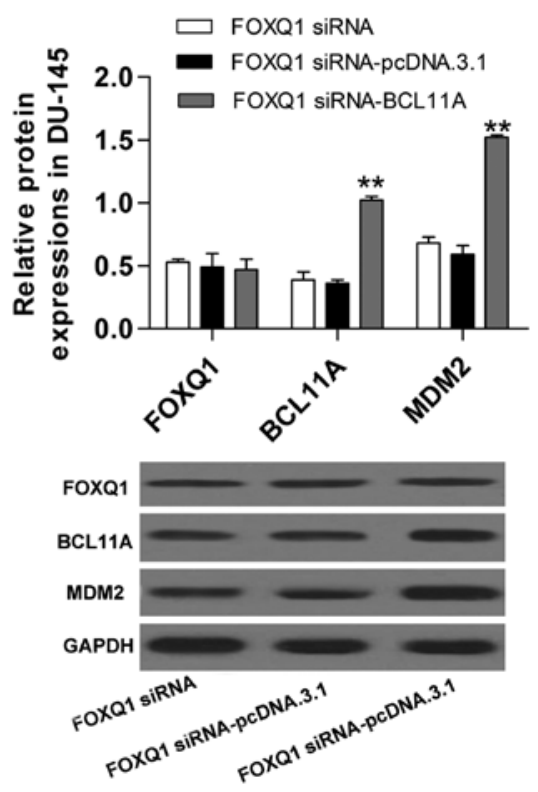

Figure 7. Protein expression of genes in prostate cancer cell lines with co-transfection. The protein expression of FOXQ1, BCL11A and MDM2 were detected by western blot analysis in PC-3 (A) and DU-145 (B) cell lines. FOXQ1 siRNA-pcDNA.3.1, cells co-transfected with FOXQ1 siRNA and pcDNA.3.1/myc-His(-) Avector; FOXQ1 siRNA-pcDNA.3.1, cells co-transfected with FOXQ1 siRNA and pcDNA.3.1-BCL11A. ${ }^{* *} \mathrm{P}<0.01$ vs. the FOXQ1 siRNA-pcDNA.3.1 group.

A

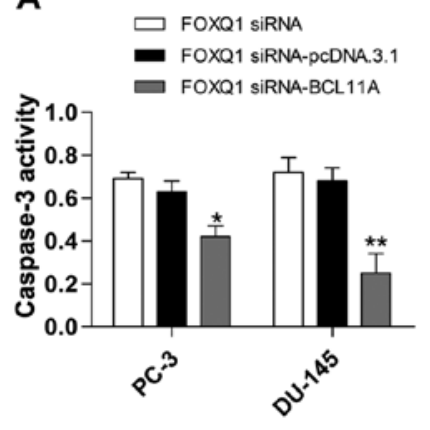

B

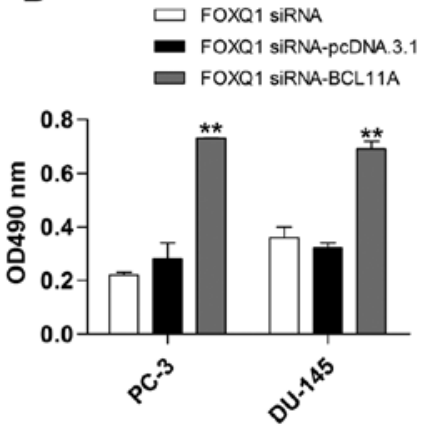

C

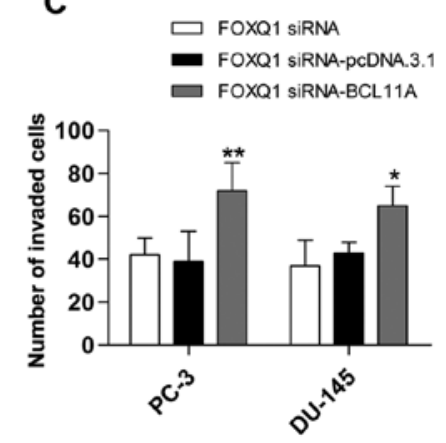

Figure 8. Effects of co-transfection on apoptosis, proliferation and invasion in prostate cancer cell lines. Apoptosis (A), proliferation (B) and invasion (C) were measured using caspase- 3 activity detection, the MTT assay and Transwell invasion assay, respectively. " $\mathrm{P}<0.05$ and ${ }^{* *} \mathrm{P}<0.01 \mathrm{vs.}$ the FOXQ1 siRNApcDNA.3.1 group. 
breast cancer, and FOXQ1 inhibition has an inhibitory effect on tumor growth and metastasis in bladder cancer and nasopharyngeal carcinoma $(22,40,41)$. It has also been demonstrated that FOXQ1 overexpression indicates a poor prognosis in NSCLC (4). However, whether FOXQ1 plays an important role in prostate cancer remained unclear. In this study, we found that FOXQ1 is upregulated in tumor tissues and cell lines, and suppression of it can, in addition to constraining proliferation, invasion and EMT, induce apoptosis in prostate cancer cells. The foundation in our study is in line with previous studies in other cancers, further confirming its oncogenic function and providing a novel target in the prevention and therapy for this disease.

BCL11A is a transcription factor with a Krüppel zincfinger motif, and the expression of BCL11A is required for not only the development pre-B-cells as well as thymocyte maturity, but also immunoglobulin switching (42-44). Moreover, BCL11A has been related to cancer. It is predicated that BCL11A has a positive role in cancers such as NSCLC, acute lymphoblastic leukemia, triple-negative breast cancer, squamous cell carcinoma as well as large cell carcinoma $(45,46)$. Additionally, studies have shown that FOXQ1 has an effect on the expression of BCL11A in colorectal cancer (21). Thus, we speculated that FOXQ1 facilitates the expression of BCL11A in prostate cancer. As expected, inhibition of FOXQ1 had a significant repressive influence on BCL11A expression in our research. Furthermore, a recent study showed that BCL11A deficient B cells exhibit reduced expression of MDM2 (25).

MDM2 exerts oncogenic functions in many cancers mainly via the inhibition of the tumor suppressor p53 (47). MDM2 gene mutation or abnormal expression leads to the destruction of the physiological balance, and is associated with cell transformation and tumorigenesis (48). Targeting the p53-MDM2 interaction has been considered as a method of treating cancer, includes the inhibition of MDM2 expression by antisense oligonucleotides (49). Studies have reported that prostate cancer is characterized by the overexpression of MDM2, and suppression of MDM2 has been reported as an experimental therapy for prostate cancer (50). Additionally, it has been found that MDM2 promotes EMT activity, whereas MDM2 inhibition constrains EMT and reduces the migratory potential of breast cancer cells (51). In our study, we found that silencing FOXQ1 exerted an inhibitory function on MDM2, while overexpression of BCL11A reversed the reduction in MDM2 induced by FOXQ1 downregulation in prostate cancer cells. Further data demonstrated that an increase in MDM2 enhanced EMT activity and cell invasion and suppressed apoptosis in prostate cancer cells, similar to the findings of Zhang et al (50). These results confirm that MDM2 is regulated by FOXQ1 via BCL11A and further has an influence on proliferation and apoptosis in prostate cancer cells.

Taken together, these data indicate that FOXQ1 acts as an oncogene in prostate cancer and is expressed at a high level in clinical prostate tissue and cell lines. Moreover, FOXQ1 inhibition constrains MDM2 by controlling BCL11A, resulting in the suppression of proliferation and invasion, and the induction of apoptosis in prostate cancer cells. Thus, our study provides novel insight into the molecular mechanism underling prostate cancer and provides a novel theoretical basis for the therapy of prostate cancer.

\section{References}

1. Carver BS, Chapinski C, Wongvipat J, Hieronymus H, Chen Y, Chandarlapaty S, Arora VK, Le C, Koutcher J, Scher H, et al: Reciprocal feedback regulation of PI3K and androgen receptor signaling in PTEN-deficient prostate cancer. Cancer Cell 19: 575-586, 2011.

2. Rastinehad AR, Baccala AA Jr, Chung PH, Proano JM, Kruecker J, Xu S, Locklin JK, Turkbey B, Shih J, Bratslavsky G, et al: D'Amico risk stratification correlates with degree of suspicion of prostate cancer on multiparametric magnetic resonance imaging. J Urol 185: 815-820, 2011.

3. Brawley OW: Prostate cancer epidemiology in the United States. World J Urol 30: 195-200, 2012.

4. Feng J, Zhang X, Zhu H, Wang X, Ni S and Huang J: FoxQ1 overexpression influences poor prognosis in non-small cell lung cancer, associates with the phenomenon of EMT. PLoS One 7: e39937, 2012.

5. Gao M, Shih IeM and Wang TL: The role of forkhead box Q1 transcription factor in ovarian epithelial carcinomas. Int J Mol Sci 13: 13881-13893, 2012.

6. Qin J, Xu Y, Li X, Wu Y, Zhou J, Wang G and Chen L: Effects of lentiviral-mediated Foxp1 and Foxq1 RNAi on the hepatocarcinoma cell. Exp Mol Pathol 96: 1-8, 2014.

7. Zhang H, Meng F, Liu G, Zhang B, Zhu J, Wu F, Ethier SP, Miller F and Wu G: Forkhead transcription factor foxq1 promotes epithelial-mesenchymal transition and breast cancer metastasis. Cancer Res 71: 1292-1301, 2011.

8. Feuerborn A, Srivastava PK, Küffer S, Grandy WA, Sijmonsma TP, Gretz N, Brors B and Gröne HJ: The Forkhead factor FoxQ1 influences epithelial differentiation. J Cell Physiol 226: 710-719, 2011.

9. Candelario J, Chen LY, Marjoram P, Reddy S and Comai L: A filtering strategy identifies FOXQ1 as a potential effector of lamin A dysfunction. Aging (Albany, NY) 4: 567-577, 2012.

10. Liang SH, Yan XZ, Wang BL, Jin HF, Yao LP, Li YN, Chen M, Nie YZ, Wang X, Guo XG, et al: Increased expression of FOXQ1 is a prognostic marker for patients with gastric cancer. Tumour Biol 34: 2605-2609, 2013.

11. Christensen J, Bentz S, Sengstag T, Shastri VP and Anderle P: FOXQ1, a novel target of the Wnt pathway and a new marker for activation of Wnt signaling in solid tumors. PLoS One 8: e60051, 2013.

12. Xiang XJ, Deng J, Liu YW, Wan LY, Feng M, Chen J and Xiong JP: miR-1271 inhibits cell proliferation, invasion and EMT in gastric cancer by targeting FOXQ1. Cell Physiol Biochem 36: 1382-1394, 2015.

13. Yuan H, Kajiyama H, Ito S, Yoshikawa N, Hyodo T, Asano E, Hasegawa H, Maeda M, Shibata K, Hamaguchi M, et al: ALX1 induces snail expression to promote epithelial-to-mesenchymal transition and invasion of ovarian cancer cells. Cancer Res 73: 1581-1590, 2013.

14. Drasin DJ, Robin TP and Ford HL: Breast cancer epithelial-to-mesenchymal transition: Examining the functional consequences of plasticity. Breast Cancer Res 13: 226, 2011.

15. Qi X, Li J, Zhou C, Lv C and Tian M: miR-142-3p suppresses SOCS6 expression and promotes cell proliferation in nasopharyngeal carcinoma. Cell Physiol Biochem 36: 1743-1752, 2015.

16. Sun HT, Cheng SX, Tu Y, Li XH and Zhang S: FoxQ1 promotes glioma cells proliferation and migration by regulating NRXN3 expression. PLoS One 8: e55693, 2013.

17. Wang W, He S, Ji J, Huang J, Zhang S and Zhang Y: The prognostic significance of FOXQ1 oncogene overexpression in human hepatocellular carcinoma. Pathol Res Pract 209: 353-358, 2013.

18. Pei Y, Wang P, Liu H, He F and Ming L: FOXQ1 promotes esophageal cancer proliferation and metastasis by negatively modulating CDH1. Biomed Pharmacother 74: 89-94, 2015.

19. Feng J, Xu L, Ni S, Gu J, Zhu H, Wang H, Zhang S, Zhang W and Huang J: Involvement of FoxQ1 in NSCLC through regulating EMT and increasing chemosensitivity. Oncotarget 5: 9689-9702, 2014.

20. Su L, Liu X, Chai N, Lv L, Wang R, Li X, Nie Y, Shi Y and Fan D: The transcription factor FOXO4 is down-regulated and inhibits tumor proliferation and metastasis in gastric cancer. BMC Cancer 14: 378, 2014.

21. Kaneda H, Arao T, Tanaka K, Tamura D, Aomatsu K, Kudo K, Sakai K, De Velasco MA, Matsumoto K, Fujita Y, et al: FOXQ1 is overexpressed in colorectal cancer and enhances tumorigenicity and tumor growth. Cancer Res 70: 2053-2063, 2010. 
22. Zhu Z, Zhu Z, Pang Z, Xing Y, Wan F, Lan D and Wang H: Short hairpin RNA targeting FOXQ1 inhibits invasion and metastasis via the reversal of epithelial-mesenchymal transition in bladder cancer. Int J Oncol 42: 1271-1278, 2013.

23. Zhang J, Li W, Dai S, Tai X, Jia J and Guo X: FOXQ1 is overexpressed in laryngeal carcinoma and affects cell growth, cell cycle progression and cell invasion. Oncol Lett 10: 2499-2504, 2015.

24. Kutlar A, Ataga K, Reid M, Vichinsky EP, Neumayr L, Blair-Britt L, Labotka R, Glass J, Keefer JR, Wargin WA, et al: A phase $1 / 2$ trial of HQK-1001, an oral fetal globin inducer, in sickle cell disease. Am J Hematol 87: 1017-1021, 2012.

25. Yu Y, Wang J, Khaled W, Burke S, Li P, Chen X, Yang W, Jenkins NA, Copeland NG, Zhang S, et al: Bcll1a is essential for lymphoid development and negatively regulates p53. J Exp Med 209: 2467-2483, 2012.

26. Staszewski O, Baker RE, Ucher AJ, Martier R, Stavnezer J and Guikema JE: Activation-induced cytidine deaminase induces reproducible DNA breaks at many non-Ig loci in activated B cells. Mol Cell 41: 232-242, 2011.

27. Tao H, Ma X, Su G, Yin J, Xie X, Hu C, Chen Z, Tan D, Xu Z, Zheng Y, et al: BCL11A expression in acute myeloid leukemia. Leuk Res 41: 71-75, 2016.

28. Jiang BY, Zhang XC, Su J, Meng W, Yang XN, Yang JJ, Zhou Q, Chen ZY, Chen ZH, Xie Z, et al: BCL11A overexpression predicts survival and relapse in non-small cell lung cancer and is modulated by microRNA-30a and gene amplification. Mol Cancer 12: 61, 2013.

29. Wade M, Li YC and Wahl GM: MDM2, MDMX and p53 in oncogenesis and cancer therapy. Nat Rev Cancer 13: 83-96, 2013.

30. Li T, Kon N, Jiang L, Tan M, Ludwig T, Zhao Y, Baer R and $\mathrm{Gu} \mathrm{W:} \mathrm{Tumor} \mathrm{suppression} \mathrm{in} \mathrm{the} \mathrm{absence} \mathrm{of} \mathrm{p53-mediated}$ cell-cycle arrest, apoptosis, and senescence. Cell 149: 1269-1283, 2012.

31. Wan Y, Wu W, Yin Z, Guan P and Zhou B: MDM2 SNP309, gene-gene interaction, and tumor susceptibility: An updated meta-analysis. BMC Cancer 11: 208, 2011.

32. Zhang J, Sun Q, Zhang Z, Ge S, Han ZG and Chen WT: Loss of microRNA-143/145 disturbs cellular growth and apoptosis of human epithelial cancers by impairing the MDM2-p53 feedback loop. Oncogene 32: 61-69, 2013.

33. Li Q and Lozano G: Molecular pathways: Targeting Mdm2 and Mdm4 in cancer therapy. Clin Cancer Res 19: 34-41, 2013.

34. Zhang YY and Zhou LM: Sirt3 inhibits hepatocellular carcinoma cell growth through reducing Mdm2-mediated p53 degradation. Biochem Biophys Res Commun 423: 26-31, 2012.

35. Tovar C, Higgins B, Kolinsky K, Xia M, Packman K, Heimbrook DC and Vassilev LT: MDM2 antagonists boost antitumor effect of androgen withdrawal: Implications for therapy of prostate cancer. Mol Cancer 10: 49, 2011

36. Welsh JB, Sapinoso LM, Su AI, Kern SG, Wang-Rodriguez J, Moskaluk CA, Frierson HF Jr and Hampton GM: Analysis of gene expression identifies candidate markers and pharmacological targets in prostate cancer. Cancer Res 61: 5974-5978, 2001.

37. Shiozawa Y, Pedersen EA, Havens AM, Jung Y, Mishra A, Joseph J, Kim JK, Patel LR, Ying C, Ziegler AM, et al: Human prostate cancer metastases target the hematopoietic stem cell niche to establish footholds in mouse bone marrow. J Clin Invest 121: 1298-1312, 2011.
38. Xia L, Huang W, Tian D, Zhang L, Qi X, Chen Z, Shang X, Nie Y and Wu K: Forkhead box Q1 promotes hepatocellular carcinoma metastasis by transactivating ZEB2 and VersicanV1 expression. Hepatology 59: 958-973, 2014.

39. Bao B, Azmi AS, Aboukameel A, Ahmad A, Bolling-Fischer A, Sethi S, Ali S, Li Y, Kong D, Banerjee S, et al: Pancreatic cancer stem-like cells display aggressive behavior mediated via activation of FoxQ1. J Biol Chem 289: 14520-14533, 2014.

40. Qiao Y, Jiang X, Lee ST, Karuturi RK, Hooi SC and Yu Q: FOXQ1 regulates epithelial-mesenchymal transition in human cancers. Cancer Res 71: 3076-3086, 2011.

41. Peng XH, Huang HR, Lu J, Liu X, Zhao FP, Zhang B, Lin SX, Wang L, Chen $\mathrm{HH}, \mathrm{Xu} X$, et al: miR-124 suppresses tumor growth and metastasis by targeting Foxq1 in nasopharyngeal carcinoma. Mol Cancer 13: 186, 2014.

42. Costa FC, Fedosyuk H, Neades R, de Los Rios JB, Barbas CF III and Peterson KR: Induction of fetal hemoglobin in vivo mediated by a synthetic $\gamma$-globin zinc finger activator. Anemia 2012: 507894, 2012

43. Esteghamat F, Gillemans N, Bilic I, van den Akker E, Cantù I, van Gent $T$, Klingmüller $U$, van Lom $K$, von Lindern $M$, Grosveld F, et al: Erythropoiesis and globin switching in compound Klf1:Bcl11a mutant mice. Blood 121: 2553-2562, 2013.

44. Liu P, Keller JR, Ortiz M, Tessarollo L, Rachel RA, Nakamura T, Jenkins NA and Copeland NG: Bcl11a is essential for normal lymphoid development. Nat Immunol 4: 525-532, 2003.

45. Kadoch C, Hargreaves DC, Hodges C, Elias L, Ho L, Ranish J and Crabtree GR: Proteomic and bioinformatic analysis of mammalian SWI/SNF complexes identifies extensive roles in human malignancy. Nat Genet 45: 592-601, 2013.

46. Khaled WT, Choon Lee S, Stingl J, Chen X, Raza Ali H, Rueda OM, Hadi F, Wang J, Yu Y, Chin SF, et al: BCL11A is a triple-negative breast cancer gene with critical functions in stem and progenitor cells. Nat Commun 6: 5987, 2015.

47. Bond GL, Hu W, Bond EE, Robins H, Lutzker SG, Arva NC, Bargonetti J, Bartel F, Taubert H, Wuerl P, et al: A single nucleotide polymorphism in the MDM2 promoter attenuates the p53 tumor suppressor pathway and accelerates tumor formation in humans. Cell 119: 591-602, 2004.

48. Jones SN, Hancock AR, Vogel H, Donehower LA and Bradley A: Overexpression of Mdm2 in mice reveals a p53-independent role for Mdm2 in tumorigenesis. Proc Natl Acad Sci USA 95: 15608-15612, 1998.

49. Klein $\mathrm{C}$ and Vassilev LT: Targeting the p53-MDM2 interaction to treat cancer. Br J Cancer 91: 1415-1419, 2004.

50. Zhang Z, Li M, Wang H, Agrawal S and Zhang R: Antisense therapy targeting MDM2 oncogene in prostate cancer: Effects on proliferation, apoptosis, multiple gene expression, and chemotherapy. Proc Natl Acad Sci USA 100: 11636-11641, 2003.

51. Wang W, Zhang X, Qin JJ, Voruganti S, Nag SA, Wang MH, Wang $H$ and Zhang $R$ : Natural product ginsenoside 25-OCH3-PPD inhibits breast cancer growth and metastasis through down-regulating MDM2. PLoS One 7: e41586, 2012. 